ARTÍCULO DE REVISIÓN

\title{
Hidradenitis supurativa
}

\section{Hidradenitis suppurativa}

\author{
Delfina G. Villanueva-Quintero* y Noel E. Luna-Romero \\ Departamento de investigación, Grupo Clínico CATEI SC, Centro de Atención en Enfermedades Inflamatorias, Guadalajara, Jalisco, México
}

\begin{abstract}
Resumen
La hidradenitis supurativa es una enfermedad cutánea crónica, inflamatoria y recurrente de la unidad pilosebácea caracterizada por la aparición de nódulos dolorosos, abscesos, trayectos fistulosos, cicatrices fibrosas y cambios de coloración de la piel. Se localiza principalmente en zonas de pliegues, como las axilas, la región submamaria y las ingles, y además puede afectar las nalgas y la región perianal y genital. Suele aparecer por primera vez después de la pubertad y persistir durante muchos años, empeorando con el tiempo, por lo que puede llegar a tener un fuerte impacto en la calidad de vida, ya que a menudo se diagnostica después de una larga evolución, cuando el paciente ya presenta abscesos, fístulas o cicatrices retráctiles, debido posiblemente a la falta de visibilidad de la enfermedad y a la escasez de guías de tratamiento basadas en la evidencia. En la presente revisión se pretende examinar los principales aspectos de esta enfermedad con especial enfoque en su clasificación clínica, la correlación con otras enfermedades inflamatorias y la evidencia y las recomendaciones respecto a las diversas opciones terapéuticas.
\end{abstract}

Palabras clave: Hidradenitis supurativa. Hidrosadenitis.

\section{Abstract}

Hidradenitis suppurativa is a chronic, inflammatory and recurrent skin disease of the pilosebaceous unit characterized by the appearance of painful nodules, abscesses, fistulous tracts, fibrous scars, and changes in skin color. It is located mainly in areas of folds such as armpits, sub mammary region, groin area, and can also affect buttocks, perianal and genital region. It usually appears for the first time after puberty and can persist for many years, worsening over time, so it can have a strong impact on quality of life, since it is often diagnosed after a long evolution, when the patient already has abscesses, fistulas, or retractable scars, possibly due to the lack of visibility of the disease and the scarcity of evidence-based treatment guidelines. This review aims to examine the main aspects of this disease with a special focus on its clinical classification, the correlation with other inflammatory diseases, and the evidence and recommendations regarding the multiple therapeutic options.

Key words: Hidradenitis suppurativa. Hidrosadenitis.

\section{Introducción}

La hidradenitis supurativa (HS), también conocida como enfermedad de Verneuil (quien la estudió inicialmente en
1854) ${ }^{1}$, hidrosadenitis, apocrinitis o acné inverso, es una enfermedad cutánea crónica, inflamatoria y recurrente de la unidad pilosebácea en la cual se encuentra una mayor cantidad de glándulas sudoríparas apocrinas.

Correspondencia: *Delfina G. Villanueva-Quintero E-mail: draluviq @gmail.com

Fecha de recepción: 03-11-2020 Fecha de aceptación: 05-02-2021 DOI: 10.24875/IMIDS.M21000002
Disponible en internet: $26-05-2021$ Rev Mex Enferm Inflam Inmunomed. 2021;95(1):6-13 www.IMIDsMexico.com 2696-6867 / @ 2021 Permanyer. Éste es un artículo open access bajo la licencia CC BY-NC-ND (http://creativecommons.org/licenses/by-nc-nd/4.0/). 
Existe una asociación entre la HS y la enfermedad inflamatoria intestinal (EII), sobre todo cuando las lesiones se localizan en la región glútea y perianal, por lo que puede ser confundida con la variante perianal de la El| ${ }^{2-4}$.

Se caracteriza por la aparición de nódulos dolorosos, abscesos, trayectos fistulosos, cicatrices fibrosas y cambios de coloración de la piel, que se localizan principalmente en las zonas de pliegues, como las axilas, la región submamaria y las ingles, y además pueden afectar las nalgas y la región perianal y genital.

Estructuralmente se caracteriza por una inflamación primaria de la glándula apocrina con obstrucción del folículo piloso, que como consecuencia directa drena su contenido (incluyendo células epidérmicas y bacterias saprófitas) al interior del canal, provocando una reacción inflamatoria mediada por células y citocinas proinflamatorias ${ }^{5}$. Esta enfermedad merece una atención prioritaria y un diagnóstico oportuno, debido a que las lesiones llegan a tener un fuerte impacto en la calidad de vida ${ }^{6}$.

Suele aparecer por primera vez después de la pubertad y puede persistir durante muchos años, empeorando con el tiempo. A menudo se diagnostica después de una larga evolución, cuando el paciente ya presenta abscesos, fístulas o cicatrices retráctiles ${ }^{7}$, debido posiblemente a la falta de visibilidad de la enfermedad y a la escasez de guías de tratamiento basadas en la evidencia $^{5}$. En el año 2015 se publicó la guía europea para el tratamiento de la HS, que contiene recomendaciones para el uso de los tratamientos que han sido aceptados $^{8}$.

En la presente revisión se comentan los principales aspectos de esta enfermedad con especial enfoque en su clasificación clínica, la correlación con otras enfermedades inflamatorias, sobre todo la Ell, y la evidencia y las recomendaciones respecto a las diversas opciones terapéuticas existentes.

\section{Epidemiología}

Según un estudio realizado por Jemec, et al. ${ }^{6}$, la frecuencia estimada de la HS es del $1-4 \%$ de la población en estudios europeos y norteamericanos. No obstante, otros estudios más recientes estiman una prevalencia del $0.97 \%^{2} \mathrm{e}$ incluso menor $(0.13 \mathrm{y}$ $0.05 \%)^{3.9}$. Un estudio realizado en Minnesota estimó una incidencia anual de 6 casos por cada 100,000 personas entre 1968 y 2008, pasando de 4 a 10 casos por cada 100,000 personas desde el inicio hasta el final del periodo de estudio ${ }^{10}$.
En México no se conoce la incidencia exacta en la población general. En un estudio realizado en el Hospital General de México ${ }^{11}$ en 2002, la prevalencia fue de 1:846.6.

Es más común en las mujeres que en los hombres, con una relación entre $2.6: 1$ y $3.3^{12.13}$. Además, las mujeres presentan afectación principalmente en el área inframamaria (22\%) y en las ingles (93\%), y los hombres en las nalgas $(40 \%)$ y la región perianal $(51 \%)^{9,14}$. Con el aumento de la edad, puede producirse una disminución en la gravedad de los síntomas ${ }^{15,16}$.

\section{Factores de riesgo}

Los principales factores de riesgo que se han relacionado para desarrollar HS son el tabaquismo (asociado con manifestaciones más graves de $\mathrm{HS}^{12}$ ), la obesidad y la historia familiar de HS, aunque también se han implicado la presencia de biofilm, factores hormonales, irritación química y traumatismos mecánicos de repetición.

\section{Tabaquismo}

El tabaquismo es un factor desencadenante de HS debido a que la nicotina promueve la obstrucción folicular. Algunos estudios han mostrado una prevalencia del $70-88.9 \%$ de fumadores entre los pacientes ${ }^{17}$.

\section{Obesidad}

La obesidad, concretamente el índice de masa corporal (IMC), se ha asociado con el desarrollo de HS. Un estudio de casos y controles con 302 pacientes mostró que el riesgo de sufrir HS aumenta con cada incremento de unidad del IMC y con el aumento del perímetro abdominal ${ }^{18}$.

\section{Historia familiar}

Entre el $30 \%$ y el $40 \%$ de los pacientes tienen familiares con HS. El patrón de herencia observado es autosómico dominante, con una penetración variable ${ }^{16}$.

\section{Otros factores}

Parece que otros factores, como la irritación química o mecánica, pueden agravar los síntomas, aunque no se dispone de estudios que lo confirmen ${ }^{4}$. 


\section{Cuadro clínico}

La HS se manifiesta como una inflamación recurrente en zonas de pliegues (axilas, ingles, submamaria), en la región perianal, las nalgas o el perineo, o en cualquier parte de la superficie cutánea donde haya glándulas apocrinas.

La manifestación inicial, en la mayoría de los casos, suelen ser macrocomedones y obstrucción (Fig. 1); posteriormente se observan nódulos (que pueden ser inflamatorios o no), abscesos (Fig. 2), trayectos fistulosos por la conexión entre estos, y por último cicatrices fibrosas (Fig. 3).

Los principales signos y síntomas de la HS dependen de su estadio evolutivo y de su gravedad. Las lesiones son dolorosas y crónicas, y la resolución de los abscesos suele dejar una cicatrización fibrosa y retráctill ${ }^{4}$.

\section{Diagnóstico y presentación clínica}

El diagnóstico de HS se realiza principalmente basándose en su presentación clínica característica. Son criterios para el diagnóstico la presencia de lesiones típicas, la topografía específica, la cronicidad y la recurrencia ${ }^{4}$. Se considera que un paciente tiene HS cuando refiere una historia recurrente de lesiones dolorosas o supurativas (más de dos veces en 6 meses) y presenta nódulos, tractos fistulosos, abscesos o cicatrices en las axilas, la zona genital, el perineo, la región perianal o la región submamaria (Fig. 4).

Como criterios secundarios para el diagnóstico se considera tener antecedentes familiares de la enfermedad y un frotis negativo o la presencia de microbiota normal de la piel, aunque se han encontrado bacterias que causan infecciones sobreagregadas; entre ellas, las más frecuentes son por Staphylococcus aureus, Staphylococcus epidermidis, Escherichia coli, Klebsiella, Proteus, estreptococos alfa y otros coliformes $^{1-8}$.

El principal diagnóstico diferencial de la HS en la región perianal debe realizarse con la enfermedad de Crohn con compromiso cutáneo; en las lesiones que afectan el pecho y el tronco se debe realizar con el acné, el cual presenta comedones cerrados.

Canoui-Poitrine, et al. ${ }^{18}$, en un estudio en población francesa, definieron tres fenotipos de pacientes con HS:

- Pacientes LC1 (axilo-mamaria), con mayor riesgo de cicatrización hipertrófica y cronicidad.

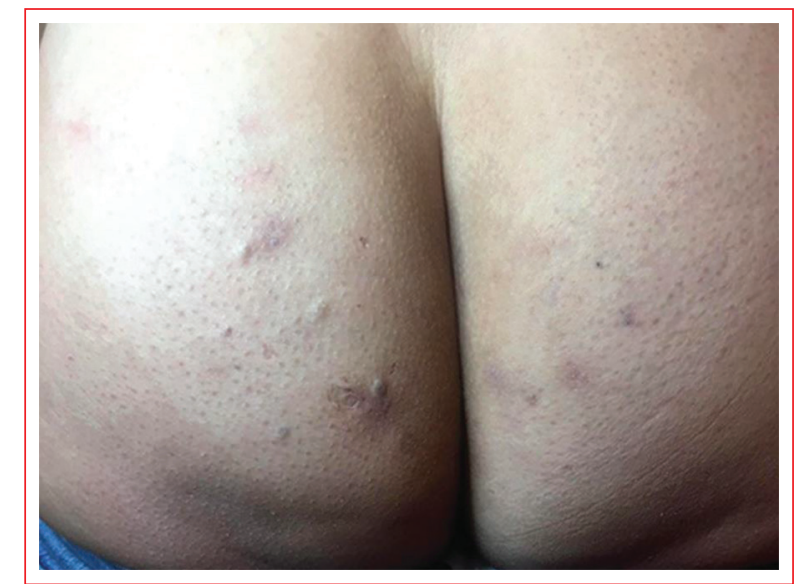

Figura 1. Hurley I. Macrocomedón Nódulo no inflamatorio Afección en los glúteos.

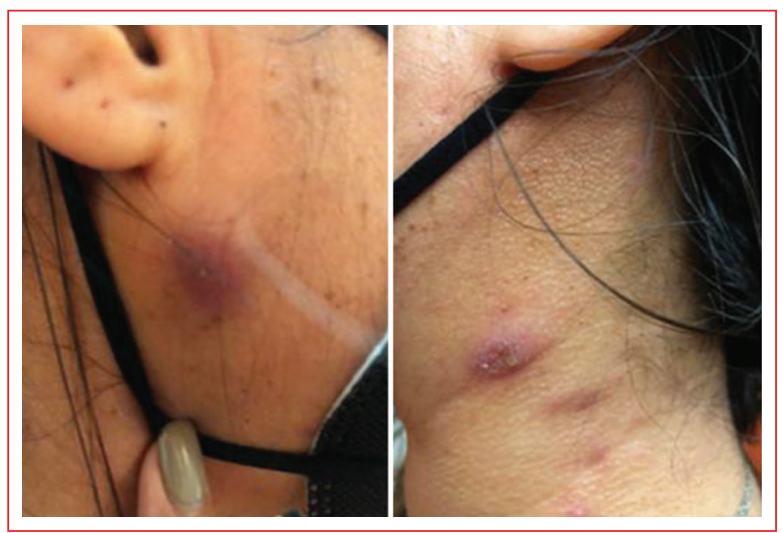

Figura 2. Hurley II. Nódulos inflamatorios en cuello, fístula drenante.

- Pacientes LC2 (folicular), en los que la enfermedad podría localizarse además en las orejas, el tórax, la espalda y las piernas, asociando lesiones foliculares (sinus pilonidal y comedones), acné grave e historia familiar, siendo varones más frecuentemente, fumadores y con HS más grave.

- Pacientes LC3 (glútea), con HS asociada a espondiloartritis o a enfermedades inflamatorias inmunomediadas, incluyendo Ell, con las lesiones eminentemente localizadas en la región glútea, a modo de pápulas y lesiones foliculares, siendo característicamente los pacientes delgados y con una HS de comportamiento menos agresivo.

Recientemente se ha sugerido un nuevo fenotipo, la HS fulminante, más habitual en varones de origen afrocaribeño y que asociaría con frecuencia síntomas reumatológicos (espondiloartritis) y anemia ${ }^{5}$. 
Tabla 1. Clasificación de Hurley

\section{Estadio Características clínicas}

I Formación de abscesos únicos o múltiples, sin trayectos fistulosos ni cicatrización

II Abscesos recurrentes, únicos o múltiples, pero ampliamente separados, con formación de trayectos fistulosos y cicatrización

III Múltiples trayectos fistulosos y abscesos interconectados, que afectan a áreas enteras

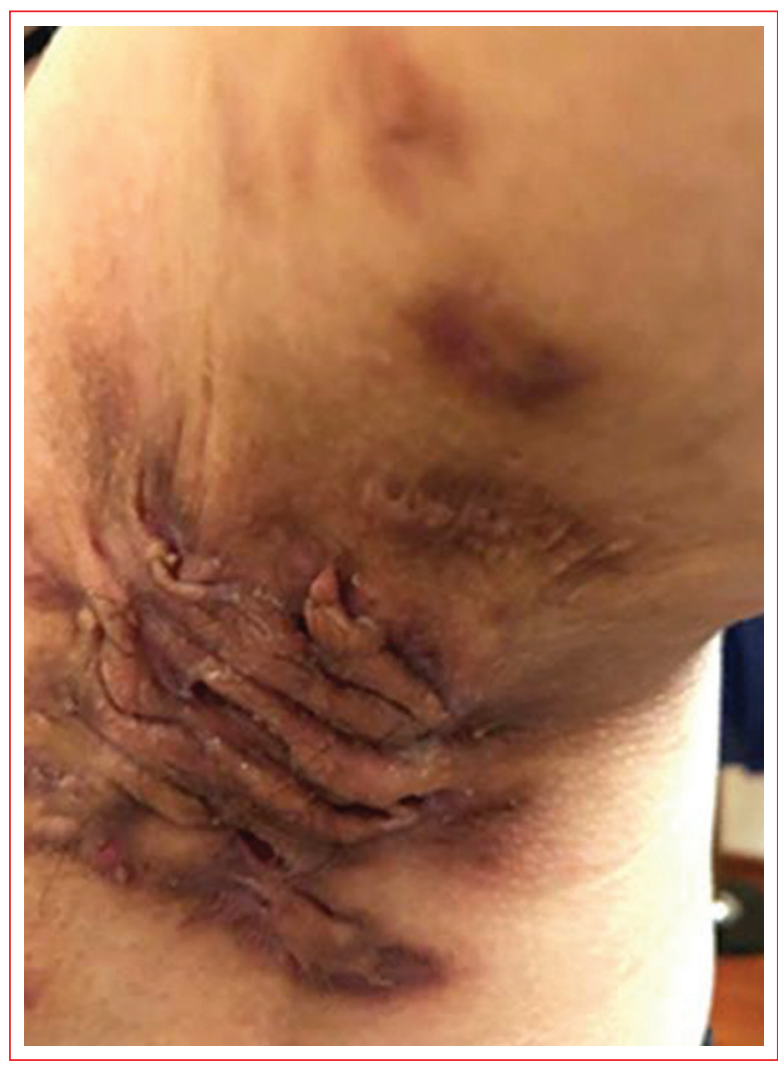

Figura 3. Hurley III.

\section{Clasificación clínica}

La clasificación de la HS según su gravedad y extensión tiene valor diagnóstico y permite seleccionar el mejor tratamiento.

La primera escala de clasificación de la gravedad de la HS fue la de Hurley ${ }^{19}$, propuesta en 1989, que sigue siendo útil en la práctica clínica. La escala de Hurley se divide en tres estadios (Tabla 1). El $68.2 \%$ de los pacientes presentan HS en estadio I, el $27.6 \% \mathrm{HS}$ en estadio II y el $3.9 \%$ HS en estadio III'.

La escala de Sartorius ${ }^{20}$ es un sistema dinámico que permite cuantificar la gravedad de la enfermedad

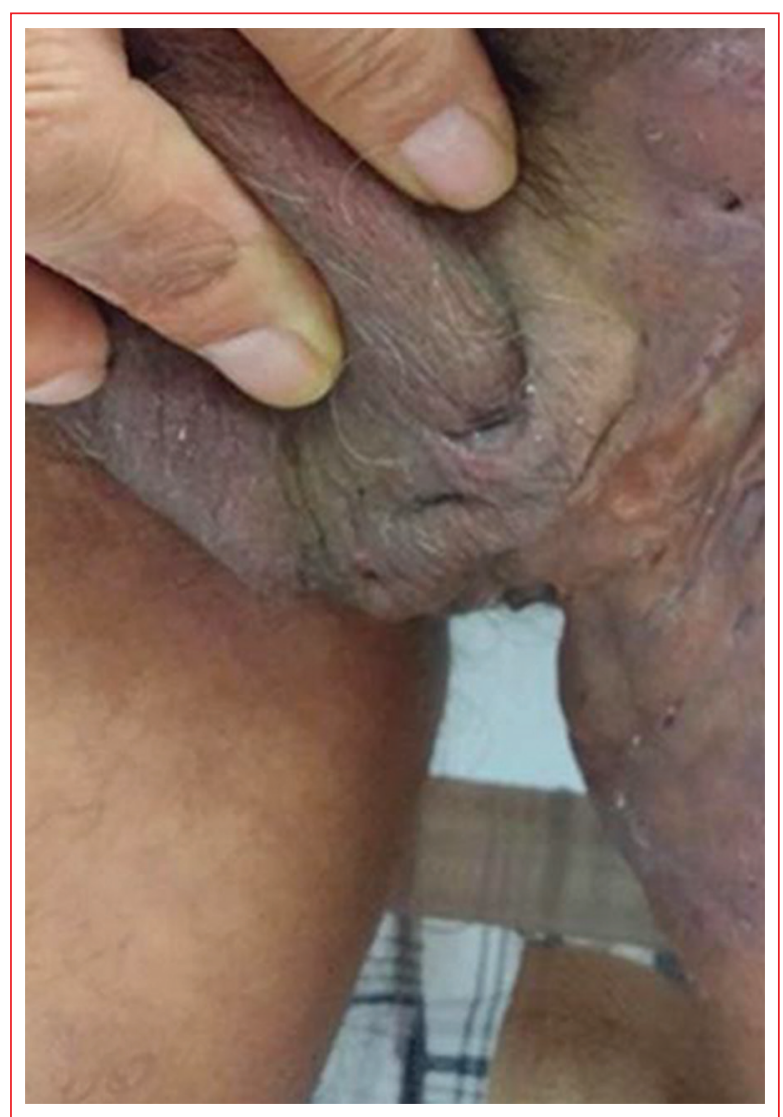

Figura 4. Hurley III.

mediante un sistema de puntuación obtenido con el recuento de las regiones anatómicas afectadas (3 puntos por región), el número y valor de las lesiones (cada nódulo recibe 1 punto y cada fístula recibe 6 puntos), la mayor distancia entre dos lesiones relevantes (< $5 \mathrm{~cm}$ : 1 punto; $5-10 \mathrm{~cm}$ : 3 puntos; $>10 \mathrm{~cm}: 9$ puntos) y la presencia de piel normal entre lesiones (sí: 0 puntos; no [Hurley III]: 9 puntos). Las distintas puntuaciones se suman y se obtiene la puntuación total del paciente ${ }^{4}$.

La evaluación global efectuada por el facultativo (Physician Global Assessment [PGA] ${ }^{21}$ ) es uno de los modelos de clasificación más utilizados 
actualmente en los ensayos clínicos para medir la mejoría clínica del paciente después del tratamiento ${ }^{4}$. Consta de seis estadios:

- Sin lesión: ausencia de nódulos inflamatorios y no inflamatorios.

- Mínimo: solo presencia de nódulos no inflamatorios.

- Leve: menos de cinco nódulos inflamatorios, o un absceso o fístula drenantes y ningún nódulo inflamatorio.

- Moderado: menos de cinco nódulos inflamatorios, o un absceso o fístula drenantes y uno o más nódulos inflamatorios, o 2-5 abscesos o fístulas drenantes y menos de 10 nódulos inflamatorios.

- Grave: 2-5 abscesos o fístulas drenantes y 10 o más nódulos inflamatorios.

- Muy grave: más de cinco abscesos o fístulas drenantes.

Otro índice de gravedad es el desarrollado por Kerdel, et al. ${ }^{23}$, que evalúa parámetros categóricos tanto objetivos como subjetivos y clasifica la enfermedad en leve (0-7 puntos), moderada (8-12) o grave (1319). La puntuación se obtiene mediante el recuento de las localizaciones, el porcentaje de superficie del cuerpo afectado, el número de lesiones eritematosas y dolorosas, el número de cambios de ropa durante las horas lectivas (como reflejo de la interferencia en las actividades de la vida diaria) y el dolor cuantificado mediante una escala análoga visual ${ }^{21}$.

La herramienta más utilizada para medir la gravedad es la clasificación de Hurley, pero hasta el momento no se dispone de parámetros específicos de mejoría y de tiempo de tratamiento para obtener el cumplimiento de las metas terapéuticas como en otras enfermedades inflamatorias cutáneas.

\section{Patogénesis}

Esta enfermedad se distingue por cuatro componentes fundamentales: a) oclusión folicular, b) inflamación, c) alteraciones inmunitarias y d) infecciones polimicrobianas $^{23}$, por lo que la atención del paciente con hidradenitis supurativa debe considerar estas características patogénicas para el manejo clínico integral.

La HS es considerada una enfermedad inflamatoria de la unidad pilosebácea, en la que hay un desequilibrio del sistema inmunitario. Las personas que la sufren tienen una predisposición genética, aunque hay factores mecánicos y hormonales, entre otros, que actúan como desencadenantes o agravantes de la enfermedad.

Existe poca información sobre el mecanismo exacto que produce la inflamación y el deterioro del tejido. La secuencia más aceptada por la que se desarrollan las lesiones consiste en hiperqueratosis y obstrucción folicular, seguidas por la dilatación del conducto hasta que tiene lugar su rotura y la salida del contenido folicular en la dermis; esto produce una respuesta del sistema inmunitario en forma de inflamación secundaria, llegada de células inflamatorias y liberación de citocinas que perpetúan el proceso de inflamación, como la interleucina $1 \mathrm{~b}$ y el factor de necrosis tumoral alfa ${ }^{2.4}$.

Por otra parte, las alteraciones del microfilm o microbiota normal se han relacionado con el desarrollo de enfermedades inflamatorias como la HS, la Ell y la psoriasis, entre otras, debido a que en los individuos genéticamente susceptibles la colonización bacteriana facilita la producción de citocinas a partir del reconocimiento de patógenos por los receptores Toll-like de los macrófagos.

\section{Comorbilidad}

La HS se ha asociado con numerosas enfermedades, entre las que se encuentran la Ell, trastornos endocrinos y metabólicos, síndromes de oclusión folicular, trastornos genéticos asociados a oclusión folicular, enfermedades articulares, enfermedades psiquiátricas, enfermedades dermatológicas, síndrome sinovitis-acné-pustulosis-hiperostosis-osteítis (SAPHO) y otros trastornos genéticos. La HS también se asocia con la presencia de neoplasias, como consecuencia de la inflamación grave crónica de la piel ${ }^{13.14}$. La HS tiene un claro impacto sobre la calidad de vida de los pacientes y es más frecuente la comorbilidad con ansiedad y depresión que en otras enfermedades inflamatorias cutáneas ${ }^{12,17}$.

La comorbilidad reportada con más frecuencia en la literatura incluye obesidad, síndrome metabólico y diabetes mellitus. Por otra parte, es relevante su asociación con enfermedades inflamatorias inmunomediadas, como espondiloartritis y Ell. Con la enfermedad de Crohn comparte características clínicas en la región perianal ${ }^{23}$.

\section{Tratamiento}

En la actualidad no hay una cura definitiva para la HS, pero los tratamientos a largo plazo permiten el control del dolor, facilitan la resolución de las lesiones y previenen las complicaciones. El tratamiento de la HS se debe adecuar a la gravedad de la enfermedad y a las características de cada paciente. Deben 
Tabla 2. Evidencia y recomendaciones de tratamiento en la hidradenitis supurativa

\begin{tabular}{|l|l|l|}
\hline Tratamiento & $\begin{array}{l}\text { Nivel de } \\
\text { evidencia }\end{array}$ & $\begin{array}{l}\text { Fuerza de } \\
\text { recomendación }\end{array}$ \\
\hline $\begin{array}{l}\text { Primera línea } \\
\text { Clindamicina tópica (leve) }\end{array}$ & IIb & B \\
$\begin{array}{l}\text { Clindamicina/rifampicina oral } \\
\text { Adalimumab }\end{array}$ & III & C \\
Tetraciclina oral & Ib & A \\
Cirugía (escisión total de las & IIb & B \\
\hline lesiones) & B \\
\hline Laser Nd YAG & IIb & A \\
\hline $\begin{array}{l}\text { Segunda línea } \\
\text { Zinc gluconato }\end{array}$ & & \\
Resorcinol & III & C \\
Corticosteroides & III & C \\
intralesionales & IV & D \\
Corticosteroides sistémicos & IV & D \\
\hline Infliximab & Ib/la & B \\
Acitretina-etretinato & III & C \\
\hline Tercera línea & & \\
Colchicina & IV & D \\
\hline Toxina botulínica & IV & D \\
\hline $\begin{array}{l}\text { Dapsotinoína } \\
\text { Ciclosporina }\end{array}$ Hormonas & IV & D \\
\hline & IV & D \\
\hline
\end{tabular}

eliminarse los factores agravantes (tabaquismo, obesidad, fricción).

Hay numerosas opciones de tratamiento con diferentes grados de evidencia y fuerza de recomendación (Tabla 2) ${ }^{8}$. Los distintos fármacos se pueden agrupar en tópicos y orales, e incluyen antibióticos (tópicos y sistémicos), antinflamatorios, terapias biológicas, retinoides y analgésicos (como terapia adyuvante). En algunas ocasiones se puede optar por el tratamiento quirúrgico, que incluye la resección de las áreas afectadas, colgajos o injertos, destechamiento y cicatrización por segunda intención (Fig. 5) 4.24.25.

Hay que tomar en consideración los niveles de evidencia y las fuerzas de recomendación:

- Niveles de evidencia:

- la: metaanálisis de estudios controlados aleatorizados.

- lb: estudios controlados aleatorizados.

- Ila: estudios controlados sin aleatorización.

- Ilb: estudios casi experimentales.

- III: estudios descriptivos no experimentales (estudios comparativos, de correlación y de casos y controles).

- IV: informes de comités de expertos, opiniones o experiencia clínica de autoridades respetadas.

- Fuerza de la recomendación:

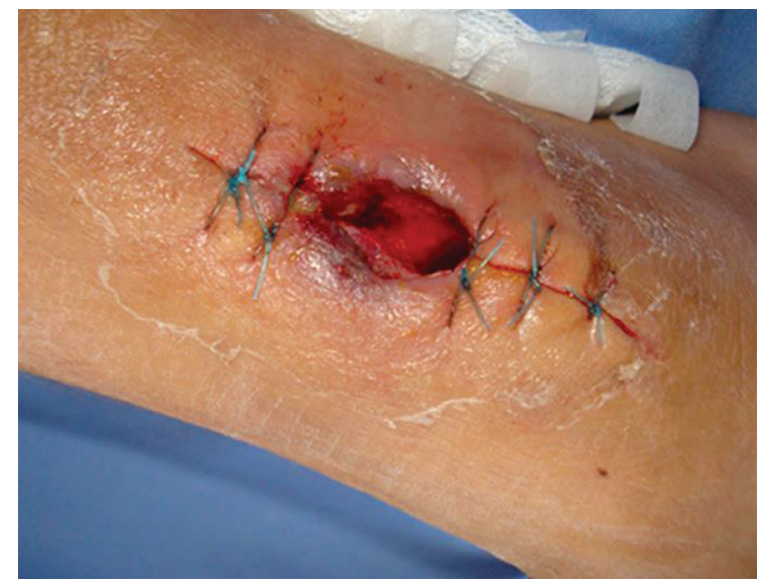

Figura 5. Tratamiento quirúrgico (destechamiento, cicatrización por segunda intención).

- A: categoría I de evidencia.

- B: categoría II de evidencia o extrapolado de la categoría I de evidencia.

- C: categoría III de evidencia o extrapolado de las categorías I o II de evidencia.

- D: categoría IV de evidencia o extrapolado de las categorías II o III de evidencia.

Teniendo en cuenta las distintas opciones terapéuticas y de acuerdo con los niveles de evidencia y las fuerzas de recomendación se elaboró un algoritmo que comprende todos los aspectos del tratamiento de la $\mathrm{HS}^{26}$.

En nuestra opinión, en estadios avanzados es necesario el tratamiento combinado con antibióticos tópicos y antiinflamatorios no esteroideos, y en estadios avanzados, si los antibióticos sistémicos combinados y el uso de retinoides no dan resultados favorables, la utilización de inhibidores del factor de necrosis tumoral (adalimumab, infliximab), incluso como monoterapia, brinda mejoría en la calidad de vida de los pacientes. Es importante la modificación de hábitos (dieta y manejo de la obesidad, eliminación del tabaquismo, ropa holgada) para que la terapia pueda lograr los objetivos de mejoría.

\section{Hidradenitis supurativa en pacientes con enfermedad inflamatoria intestinal}

Existen numerosos estudios que confirman la existencia de una asociación entre la HS y la Ell. Además, resulta evidente que ambos procesos inflamatorios guardan una serie de similitudes. 
En comparación con la población general, en un estudio de 679 casos realizado en Minnessota $^{10}$ (Estados Unidos de Norteamérica), la tasa de incidencia de HS en los pacientes con Ell fue de 8.9. Otro estudio $^{7}$, en población europea, retrospectivo de 10 años, con un total de 1260 pacientes, reveló una prevalencia significativamente mayor con respecto a la población general $(6,8-10,6 \%$ frente a $1-2 \%)$, y una mayor prevalencia, del $80 \%$, para la enfermedad de Crohn con respecto a la colitis ulcerativa. Por otra parte, en servicios de dermatología en Holanda y Bélgica $^{27}$, entre 2007 y 2015, fueron revisados los datos de 1076 pacientes con HS, 324 hombres y 752 mujeres, de los cuales el $43.9 \%$ estaban en estadio Hurley I y el $44.2 \%$ en estadio II, con un tiempo de evolución promedio de 11 años, y se encontró una prevalencia del $3.3 \%$; además, se encontró el $75 \%$ con enfermedad de Crohn y el $25 \%$ con colitis ulcerativa. Por otra parte, no se observó una mayor afección en pacientes con obesidad o una mayor gravedad de la enfermedad (como en otros reportes de la literatura). Esto indica que la prevalencia de Ell es de 4 a 8 veces más alta en las cohortes de este estudio que en la población general27.

Un metaanálisis demostró una asociación significativa entre HS y Ell (enfermedad de Crohn y colitis ulcerativa), en comparación con la población general, estadísticamente mayor para la enfermedad de Crohn que para la colitis ulcerativa. Por ello, los autores sugieren un tamizaje de los pacientes con HS en búsqueda de síntomas y signos de Ell, sobre todo en presencia de enfermedad perineal ${ }^{28}$.

En algunas revisiones ${ }^{27.28}$, la presentación glútea o perineal de la HS se ha relacionado con una mayor frecuencia de asociación a Ell, representando el 26\% del total con respecto a las otras topografías.

Estudios posteriores han confirmado la mayor tendencia del paciente con Ell a asociar HS con afectación perianal $(73 \%)$, así como en las áreas axilar (53\%) e inguinal $(47 \%)^{2.28}$.

Actualmente, la enfermedad de Crohn se considera una enfermedad asociada a HS. Puede ser necesaria la biopsia o la colonoscopia, aun en ausencia de síntomas digestivos. Se han observado casos con enfermedad de Crohn que desarrollan posteriormente HS. Los inhibidores del factor de necrosis tumoral han demostrado eficacia en ambos cuadros clínicos, lo que argumenta que estas enfermedades compartan citocinas proinflamatorias en su patogénesis ${ }^{22}$.

El metaanálisis de Phan, et al. ${ }^{28}$ reveló una asociación significativa entre ambas enfermedades (1.0 vs.
0.5\%; odds ratio [OR]: 2.05; intervalo de confianza del 95\% [IC95\%]: 1.47-2.86; $p<0.0001$ ), y en un subgrupo de análisis, de acuerdo con el tipo de Ell, encontró una mayor prevalencia de HS en la enfermedad de Crohn, en comparación con controles sin HS (OR: 1.98; IC95\%: 1.17-3.32; $p=0.01$ ). En cuanto a la colitis ulcerativa, también halló una asociación importante, en comparación con los controles (OR: 1.65; IC95\%: 1.20-2.27).

El diagnóstico de HS en un paciente con Ell es clínico, debido a que en el estudio histológico de ambas enfermedades se observan procesos granulomatosos neutrofílicos, pero la histopatología de la HS demuestra obstrucción de los conductos glandulares y secreción glandular apocrina por decapitación (contenido celular dentro del conducto) ${ }^{1}$.

La asociación de la HS a la Ell es en realidad más frecuente de lo que se había pensado. Así, el carácter multifactorial, la existencia de interacción de factores anatómicos, genéticos y ambientales, como el tabaco, la obesidad, el carácter crónico de la enfermedad, el comportamiento recidivante, el desarrollo de fístulas, así como la falta de respuesta a algunos tratamientos sistémicos y las tasas de respuesta a tratamientos dirigidos contra el factor de necrosis tumoral, permiten establecer la hipótesis de que la Ell, principalmente la enfermedad de Crohn, y la HS son enfermedades que forman parte de un espectro clínico-patogénico común.

Los pacientes con HS moderada y grave, al igual que ocurre en los pacientes con Ell, suponen un reto terapéutico ya no solo en el control de un brote ocasional, sino en el mantenimiento libre de enfermedad.

Una exploración de los pliegues axilar e inguinal, así como de la región glútea, permitirá detectar precozmente aquellos casos que asocien HS a la Ell.

\section{Conclusiones}

La HS es una enfermedad que tiene un gran impacto en la calidad de vida, no solo por el aspecto, sino también por la sintomatología y las limitaciones en la vida diaria del individuo que la padece.

Es importante una adecuada anamnesis de los pacientes con HS para poder establecer una asociación con otras enfermedades inflamatorias y dar un tratamiento oportuno.

Es necesario, además, el perfilamiento de los pacientes para seleccionar el tipo de terapia, de acuerdo con las necesidades propias y la comorbilidad, así como la elaboración de guías que avalen los fármacos con mayor eficacia para la HS asociada a otras enfermedades. 


\section{Agradecimientos}

A la Dra. Citlali Ramírez, por su colaboración en la estructura de este artículo.

\section{Conflicto de intereses}

Los autores declaran que no existen conflictos de intereses.

\section{Responsabilidades éticas}

Protección de personas y animales. Los autores declaran que para esta investigación no se han realizado experimentos en seres humanos ni en animales.

Confidencialidad de los datos. Los autores declaran que han seguido los protocolos de su centro de trabajo sobre la publicación de datos de pacientes.

Derecho a la privacidad y consentimiento informado. Los autores han obtenido el consentimiento informado de los pacientes y/o sujetos referidos en el artículo.

\section{Bibliografía}

1. Martorell A, García-Martínez FJ, Jiménez-Gallo D, Pascual JC, Pereyra-Rodriguez J, Salgado L, et al. An update on hidradenitis suppurativa (Part I): Epidemiology, clinical aspects, and definition of disease severity. Actas Dermosifiliogr. 2015;106:716-24.

2. Martorell A. Asociación entre hidradenitis supurativa y enfermedad inflamatoria intestinal. Enferm Inflam Intest Dia. 2016;15(2):57-64.

3. Crowley JJ, Mekkes JR, Zouboulis CC, Scheinfeld N, Kimball A, Sundaram $M$, et al. Association of hidradenitis suppurativa disease severity with increased risk for systemic comorbidities. Br J Dermatol. 2014;171:1561.

4. Villanueva D, Rocha J, Rivera C. Guía de diagnóstico y tratamiento de la hidradenitis supurativa. Permanyer México; 2016.

5. Jemec GBE. Clinical practice. Hidradenitis suppurativa. N Engl J Med.2012;366:158-64.

6. Jemec GB, Heidenheim M, Nielsen $\mathrm{NH}$. The prevalence of hidradenitis suppurativa and its potential precursor lesions. J Am Acad Dermatol. 1996;35:191-4.

7. Dufour DN, Emtestam L, Jemec GB. Hidradenitis suppurativa: a common and burdensome, yet under-recognised, inflammatory skin disease. Postgrad Med J.2014;90:216-21, quiz 220. Disponible en: http://www.pubmedcentral.nih.gov/articlerender.fcgi?artid=3963556\&tool=pmcentrez\&rendertype=Abstract

8. Zouboulis CC, Desai N, Emtestam L, Hunger RE, loannides D, Juhász I, et al. European $\mathrm{S} 1$ guideline for the treatment of hidradenitis suppurativa/ acne inversa. J Eur Acad Dermatol Venereol. 2015;29:619-44.
9. Canoui-Poitrine F, Le Thuaut A, Revuz JE, Viallette C, Gabison G, Poli $F$, et al. Identification of three hidradenitis suppurativa phenotypes: latent class analysis of a cross-sectional study. J Invest Dermatol. 2013;133:1506-11.

10. Shahi V, Alikhan A, Vazquez B, G, Weaver A, L, Davis M, D: Prevalence of Hidradenitis Suppurativa: A Population-Based Study in Olmsted County, Minnesota. Dermatology 2014;229:154-158.

11. Charúa-Guindic L, Maldonado-Barrón R, Avendaño-Espinosa O. Hidradenitis supurativa. Cir Cir. 2006;74:249-55.

12. Shavit E, Dreiher J, Freud T, Halevy S, Vinker S, Cohen AD. Psychiatric comorbidities in 3207 patients with hidradenitis suppurativa. J Eur Acad Dermatol Venereol. 2015;29(2):371-6.

13. Van der Zee HH, Van der Woude CJ, Florencia EF, Prens EP. Hidradenitis suppurativa and inflammatory bowel disease: are they associated? Results of a pilot study. Br J Dermatol. 2010;162:1957.

14. Scheinfeld N. Diseases associated with hidradenitis suppurativa: part 2 of a series on hidradenitis. Dermatol Online J. 2013;19:18558.

15. Gold DA, Reeder VJ, Mahan MG, Hamzavi IH. The prevalence of metabolic syndrome in patients with hidradenitis suppurativa. J Am Acad Dermatol.2014;70(4):699-703.

16. Kohorst JJ, Kimball AB, Davis M. Systemic associations of hidradenitis suppurativa. J Am Acad Dermatol. 2015;73:S27-35.

17. Dufour DN, Emtestam L, Jemec GB. Hidradenitis suppurativa: a common and burdensome, yet under-recognised, inflammatory skin disease. Postgrad Med J. 2014;90(1062):216-21.

18. Canoui-Poitrine F, Revuz JE, Wolkenstein P, Gabison G, Pouget F, Poli F, et al. Clinical characteristics of a series of 302 French patients with hidradenitis suppurativa, with an analysis of factors associated with disease severity. J Am Acad Dermatol. 2009;61:51-7.

19. Hurley HJ. Axillary hyperhidrosis, apocrine bromhidrosis, hidradenitis suppurativa and familial benign pemphigus: surgical approach. In: Roenigk RK, Roenigk HH, Jr, eds. Dermatologic surgery: principles and practice. 2nd ed. New York: Marcel Dekker, 1996:623-45.

20. Sartorius K, Emtestam L, Jemec GB, Lapins J. Objective scoring of hidradenitis suppurativa reflecting the role of tobacco smoking and obesity. Br J Dermatol. 2009 Oct;161(4):831-9.

21. Kimball AB, Kerdel F, Adams D, Mrowietz U, Gelfand JM, Gniadecki R, et al. Adalimumab for the treatment of moderate to severe hidradenitis suppurativa: a parallel randomized trial. Ann Intern Med. 2012; 157(12):846-55.

22. Grant A, Gonzalez T, Montgomery MO, Cardenas V, Kerdel FA. Infliximab therapy for patients with moderate to severe hidradenitis suppurativa: a randomized, double-blind, placebo-controlled crossover trial. J Am Acad Dermatol. 2010;62(2):205-17.

23. Estrada AL, Guzmán AR, García HH, Martínez OJ, Morales MY, Rocha RJ, et al. Consenso mexicano en el manejo clínico de la hidradenitis supurativa. Revisión sistemática. Med Int Mex. 2019; 35(4):564-84.

24. Revuz JE, Canoui-Poitrine F, Wolkenstein P, Viallette C, Gabison G, Pouget $F$, et al. Prevalence and factors associated with hidradenitis suppurativa: results from two case-control studies. J Am Acad Dermatol. 2008;59:596-601.

25. Van der Zee HH, Laman JD, de Ruiter L, Dik WA, Prens EP. Adalimumab (antitumour necrosis factor- $\alpha$ ) treatment of hidradenitis suppurativa ameliorates skin inflammation: an in situ and ex vivo study. BrJDermatol.2012;166(2):298-305.

26. Guyatt GH, Oxman AD, Vist GE, Kunz R, Falck-Ytter Y, Alonso-Coello $P$, et al. GRADE: an emerging consensus on rating quality of evidence and strength of recommendations. BMJ. 2008;336(7650):924-6.

27. Deckers IE, Benhadou F, Koldijk MJ, Del Marmol V, Horváth B, Boer J, et al. Inflammatory bowel disease is associated with hidradenitis suppurativa: results from a multicenter cross-sectional study. J Am Acad Dermatol. 2017;76(1):49-53.

28. Phan K, Tatian A, Woods J, Cains G, Frew JW. Prevalence of inflammatory bowel disease (IBD) in hidradenitis suppurativa (HS): systematic review and adjusted meta-analysis. Int J Dermatol. 2020;59(2):221-8. 